\title{
Treatment outcomes for hepatoblastoma children with pulmonary metastasis and extrapulmonary involvement: experience of 36 cases at a single institution
}

\author{
Hui-Min Hu\#, Wei-Ling Zhang", Yi-Zhuo Wang, Yi Zhang, You Yi, Fan Li, Tian Zhi, Dong-Sheng Huang \\ Department of Pediatrics, Beijing Tongren Hospital, Capital Medical University, Beijing, China \\ Contributions: (I) Conception and design: DS Huang; (II) Administrative support: DS Huang; (III) Provision of study materials or patients: YZ \\ Wang, Y Zhang; (IV) Collection and assembly of data: HM Hu, WL Zhang; (V) Data analysis and interpretation: Y Yi, T Zhi, F Li; (VI) Manuscript \\ writing: All authors; (VII) Final approval of manuscript: All authors. \\ "These authors contributed equally to work as co-first authors. \\ Correspondence to: Dong-Sheng Huang. Department of Pediatrics, Beijing Tongren Hospital, Capital Medical University, West South Road 2, \\ Yizhuang Economic and Technological Development Zone, Beijing 100176, China. Email: hdsmed@163.com.
}

Background: Hepatoblastoma $(\mathrm{HB})$ was the most common primary liver malignant tumor in children. In this study, we aimed to analyze the clinical characteristics and outcome in HB children with pulmonary metastasis and extrapulmonary involvement.

Methods: This retrospective study enrolled $36 \mathrm{HB}$ children with pulmonary metastasis and extrapulmonary involvement from January 2010 to December 2017. Clinical characteristics, treatment and outcomes were collected and analyzed. Survival curves were calculated by Kaplan-Meier method.

Results: Thirty-six patients (10 females and 26 males) were recruited, with a mean onset age of 2.13 years (range, 0.33-7.83). Four (11.11\%) patients presented with single metastatic pulmonary nodules, 32 (88.89\%) patients presented with multiple metastatic nodules in both lungs. There were $10(27.78 \%)$ patients with extrahepatic abdomen involvement, 13 (36.11\%) patients with brain metastasis, and 16 (44.44\%) patients with vascular metastasis. All patients underwent liver tumorectomy and chemotherapy. The median chemotherapy cycle was 17 (range, 3-39). In addition, 19 (54.29\%) patients underwent lung metastasectomy. The patients were followed up to December 2018, with a median follow-up of 32.5 months. At the study closing date, 9 patients were alive, 24 patients had died, and 3 patients were censored. Alpha fetoprotein (AFP) level, PRETEXT stage and distant metastases had significant impact on survival time (all $\mathrm{P}<0.05$ ).

Conclusions: The common sites of extrapulmonary metastasis of HB were blood vessels, brain and extrahepatic abdominal organs. The overall prognosis of HB patients with lung metastasis and extrapulmonary involvement was poor, especially those with PRETEXT stage IV, high AFP level or distant metastases.

Keywords: Children; clinical characteristics; extrapulmonary involvement; hepatoblastoma (HB); pulmonary metastasis

Submitted Apr 20, 2020. Accepted for publication Sep 03, 2020.

doi: $10.21037 /$ tcr-20-1876

View this article at: http://dx.doi.org/10.21037/tcr-20-1876 


\section{Introduction}

Hepatoblastoma (HB) is the most common primary liver malignant tumor in children, and has a prevalence of 0.9 per million population $(1,2)$. HB usually affects children under 3 years of age, presenting as a large abdominal mass (3). The diagnosis of $\mathrm{HB}$ is initially performed based on the elevated alpha-fetoprotein (AFP) level and radiographic detection of a liver mass, and confirmed by pathological examination of samples obtained via either primary liver resection or biopsy (4). Several factors are reported to have a significant impact on prognosis, including PRETEXT stage, pathology type, AFP level at diagnosis and after chemotherapy, and distant metastasis (5-8).

In the 1970s, the main treatment for HB was single tumor resection, which leaded to a very low overall survival (OS) rate (20\% to $30 \%$ ) (9). Since the 1980 s, the comprehensive treatment of surgical combined with chemotherapy has significantly improved prognosis, and the 5 -year survival rate could reach $75 \%(10,11)$. However, patients with advanced HB usually have poor prognosis, especially those with an extensive unifocal or multifocal primary tumor or distant metastases (12). About one fifth of the patients have lung metastasis at diagnosis, and the recurrence of $\mathrm{HB}$ mostly occurred in the lung $(13,14)$. In addition, reports on the prognosis of $\mathrm{HB}$ with extrapulmonary involvement are relatively rare $(2,15,16)$. Therefore, we aimed to retrospectively analyze the clinical characteristics and outcome in HB children with pulmonary metastasis and extrapulmonary involvement in this study and provide some valuable information for further treatment. We present the following article in accordance with the STROBE reporting checklist (available at http:// dx.doi.org/10.21037/tcr-20-1876).

\section{Methods}

\section{Patients}

We retrospectively reviewed $36 \mathrm{HB}$ patients with pulmonary metastasis and extrapulmonary involvement from January 2010 to December 2017. The inclusion criteria were as follows: (I) patients diagnosed with pulmonary metastasis and extrapulmonary involvement $\mathrm{HB}$ for the first time in our hospital; (II) patients with age $<14$ years; (III) lung enhanced computed tomographic (CT) or positron emission tomographic (PET)-CT showed that patients with metastatic nodules; (IV) patients with extrapulmonary involvement had corresponding imaging evidence. Patients who received any prior treatments were excluded. The study was conducted in accordance with the Declaration of Helsinki (as revised in 2013). The study was approved by the ethics committee of the Beijing Tongren Hospital, Capital Medical University (No.: 20180212) and informed consent was taken from all participants and their guardians.

\section{Diagnostic criteria}

The diagnosis was based on the clinical examination, operative biopsy or percutaneous needle biopsy. Histology was mainly classified as epithelial (including embryonal, macrotrabecular and fetal subtypes) or mixed type (17). HB staging (stage I-IV) was performed according to the PRETEXT staging guidelines established by the International Childhood Liver Tumor Strategy Group (SIOPEL) (18). Risk stratification (extremely low risk, low risk, intermediate risk, high risk) for HB was based on both a SIOPEL risk stratification system and a Children's Oncology Group (COG) staging system $(19,20)$.

\section{Therapy}

The study design was formulated with reference to CHIC (15), COG (21-23) and other organizations. Patients received comprehensive treatment. Patients with PRETEXT stage III and IV received pre-surgical chemotherapy, surgery and post-surgical chemotherapy. In addition, patients with PRETEXT stage II underwent complete resection and adjuvant chemotherapy. Pre-surgical chemotherapy was sustained for 3-5 cycles. Post-surgical chemotherapy was sustained for 4-6 cycles. Twenty-one to twenty-eight days were one cycle of chemotherapy. The commonly used chemotherapy regimens were VICC (vincristine+irinotecan+cyclophosphamide+cisplatin) and VIFC (vincristine+irinotecan+fluorouracil+cisplatin) (24). Serum AFP levels, blood routine test, blood biochemical routine and electrocardiogram were detected in each cycle. Primary and metastatic lesions were evaluated every 2 cycles of chemotherapy. The surgeries included hepatectomy, thrombectomy, and lung mass resection. Other treatment measures (interventional therapy, radiofrequency ablation, ultrasound focused scalpel, etc.) were selected according to the tumor condition.

\section{Analysis of therapeutic effect and follow-up}

The prognosis of all patients was indicated by OS, which 
Table 1 Demographic and clinical characteristics of included patients

\begin{tabular}{|c|c|}
\hline Characteristic & Number (n, \%) \\
\hline \multicolumn{2}{|l|}{ Gender } \\
\hline Male & $26(72.22)$ \\
\hline Female & $10(27.78)$ \\
\hline \multicolumn{2}{|l|}{ Pathology type } \\
\hline Epithelial & $62(62.27)$ \\
\hline Mixed & $36(36.73)$ \\
\hline Not clearly classified & $1(2.78)$ \\
\hline \multicolumn{2}{|l|}{ PRETEXT stage } \\
\hline Stage III & $12(33.33)$ \\
\hline Stage IV & $24(66.67)$ \\
\hline \multicolumn{2}{|l|}{ Serum AFP ( $\mu \mathrm{g} / \mathrm{L})$} \\
\hline$<100,000$ & $21(58.33)$ \\
\hline$\geq 100,000$ & $15(41.67)$ \\
\hline \multicolumn{2}{|l|}{ Pulmonary metastasis } \\
\hline Single nodule in one lung & $4(11.11)$ \\
\hline Multiple nodules in both lungs & $32(88.89)$ \\
\hline \multicolumn{2}{|l|}{ Extrapulmonary involvement } \\
\hline Blood vessels & $16(44.44)$ \\
\hline Extrahepatic abdomen & $10(27.78)$ \\
\hline Mediastinum & $3(8.33)$ \\
\hline Brain & $13(36.11)$ \\
\hline Spinal cord & $1(2.78)$ \\
\hline Bone & $4(11.11)$ \\
\hline Bone marrow & $1(2.78)$ \\
\hline
\end{tabular}

$\mathrm{HB}$, hepatoblastoma; AFP, alpha-fetoprotein.

was calculated from the day of first admission to our hospital to the time of the last follow-up or death. Censored cases were defined as patients who died from non-tumor causes. The evaluation criteria of chemotherapy efficacy were partial response (PR) and complete response (CR). $\mathrm{PR}$ was defined as a reduction of the product of the largest perpendicular diameters of all measurable lesions by more than $50 \%$. CR was defined as disappearance of all known tumor lesions. Recurrence was defined as biopsy confirmation, with clear imaging evidence and serum AFP increased 3 times continuously within 4 weeks. The patients were followed up to December 2018, with a median followup of 32.5 months.

\section{Statistical analysis}

All statistical analyses were performed by using SPSS version 21.0 (SPSS Institute, IL, USA). Quantitative data were expressed as means \pm standard deviations $(\mathrm{SD})$ and were compared using Student's $t$-test. Qualitative data were expressed as number and percentage and were compared using $\chi^{2}$ test. Survival curves were calculated by KaplanMeier method. Statistical significance was set at $\mathrm{P}<0.05$.

\section{Results}

\section{Demographic and clinical characteristics}

A total of $36 \mathrm{HB}$ patients (26 males, 10 females; median age, 2.13 years; range, $0.33-7.83$ years) with pulmonary metastasis and extrapulmonary involvement were enrolled in this retrospective study from January 2010 to December 2017. The demographic and clinical characteristics of included patients were shown in Table 1 .

Among the 36 patients, 18 (50.00\%) were epithelial subtype, 17 (47.22\%) were mixed subtype and 1 (2.78\%) was not clearly classified. There were $12(33.33 \%)$ patients with PRETEXT stage III and $24(66.67 \%)$ patients with PRETEXT stage IV. The median serum AFP level was $52,000 \mu \mathrm{g} / \mathrm{L}$ (range, 726-25,009,220). Four (11.11\%) patients presented with single metastatic pulmonary nodules, $32(88.89 \%)$ patients presented with multiple metastatic nodules in both lungs. There were 10 (27.78\%) patients with extrahepatic abdomen metastasis, 3 (8.33\%) patients with mediastinum metastasis, 13 (36.11\%) patients with brain metastasis (Figure 1), 1 (2.78\%) patients with spinal cord metastasis, $4(11.11 \%)$ patients with bone metastasis (Figure 2), 16 (44.44\%) patients with vascular metastasis (Figure 3), and 1 (2.78\%) patients with bone marrow metastasis.

\section{Treatment and outcomes}

All patients were treated with surgery and chemotherapy (Table 2). All patients underwent liver tumorectomy, of which $24(68.57 \%)$ patients underwent 1-time liver tumorectomy, 9 (25.71\%) patients underwent 2 times liver tumorectomy, and $3(8.57 \%)$ patients underwent 3 times liver tumorectomy. Otherwise, 35 (97.22\%) patients 

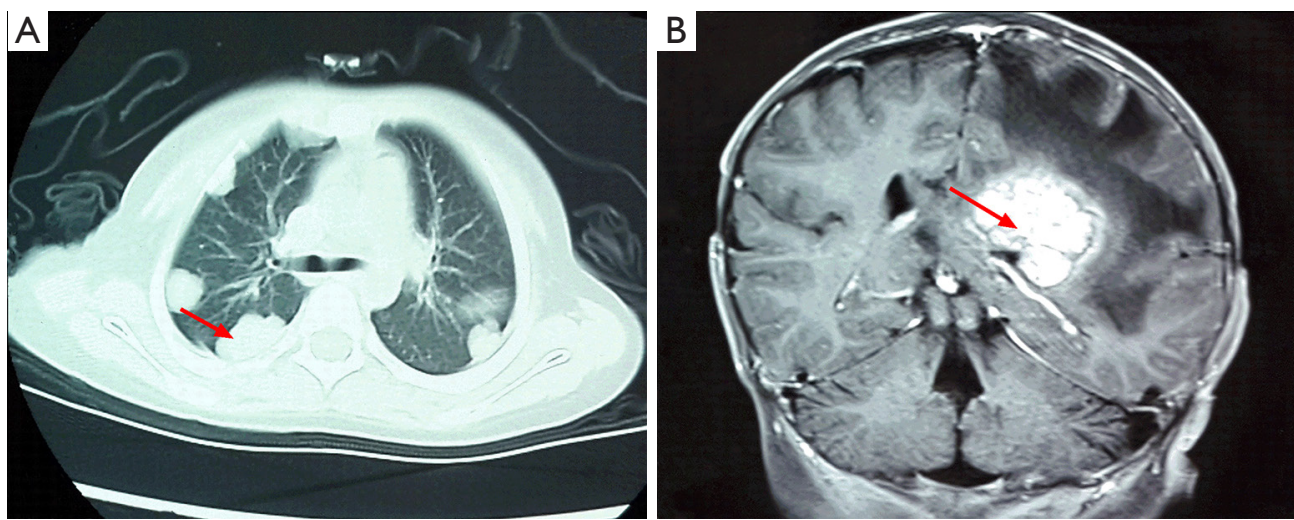

Figure 1 One hepatoblastoma children with pulmonary metastasis and brain metastasis. (A) Children presented with multiple metastatic nodules in both lungs. (B) Hepatoblastoma with left parietal lobe brain metastases.
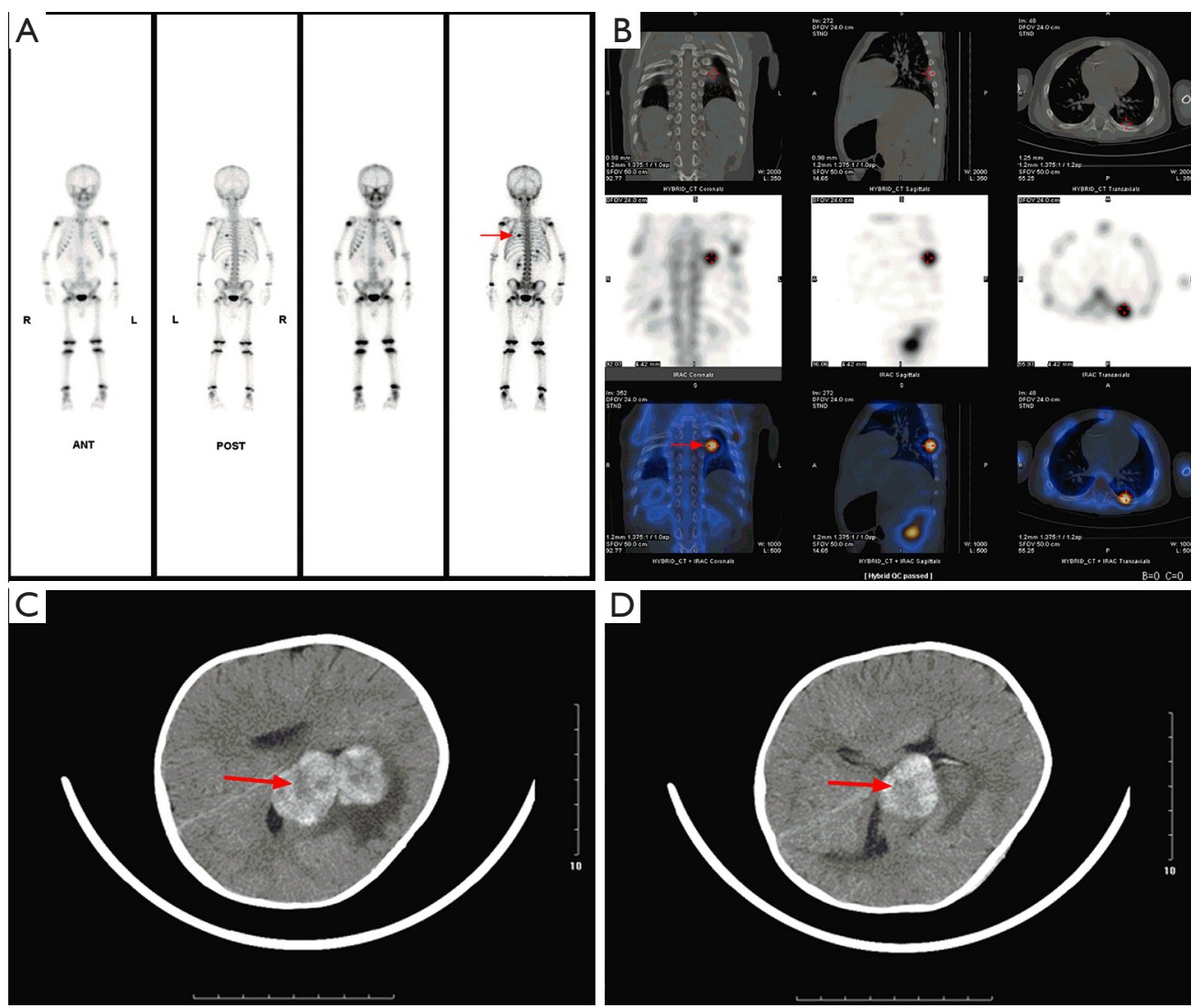

Figure 2 One hepatoblastoma children with bone metastasis and brain metastasis. (A,B) Hepatoblastoma with bone metastasis. (C,D) Hepatoblastoma with brain metastases. 

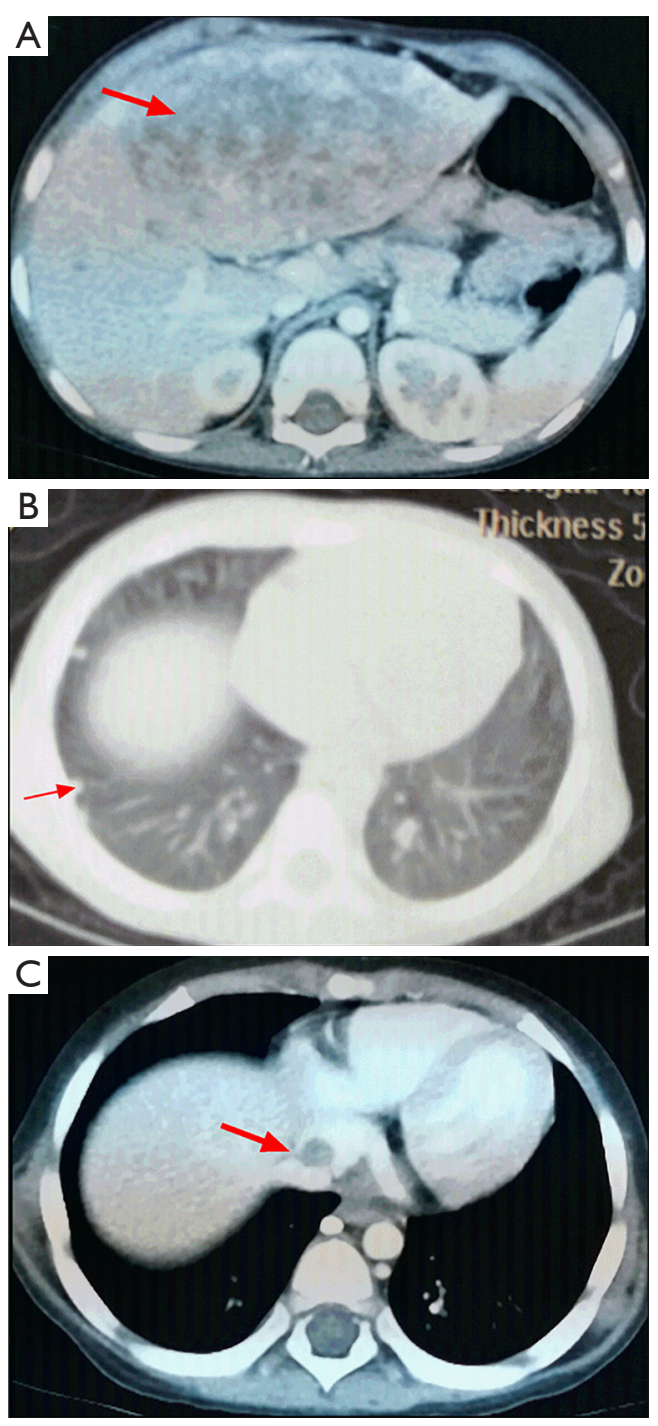

Figure 3 One hepatoblastoma children with pulmonary metastasis and right atria metastasis. (A) Tumor shadow with uneven density in the left lobe of liver. (B) Hepatoblastoma with multiple right pulmonary metastases. (C) Hepatoblastoma with right atrial metastasis.

received standardized chemotherapy for more than 6 cycles, and $1(2.78 \%)$ patient only received three cycles of chemotherapy. The median chemotherapy cycle was 17 (range, 3-39), of which pre-surgical median chemotherapy cycle was 3 (range, 0-8) and post-surgical median chemotherapy cycle was 14 (range, 0-39). In addition, $19(54.29 \%)$ patients underwent lung metastasectomy, of which $10(28.57 \%)$ patients underwent 1 time lung metastasectomy, 3 (8.57\%) patients underwent 2 times lung
Table 2 Surgery and outcomes of included patients

\begin{tabular}{|c|c|}
\hline Characteristics & Number (n, \%) \\
\hline \multicolumn{2}{|l|}{ Standard treatment } \\
\hline No & $1(2.78)$ \\
\hline Yes & $35(97.22)$ \\
\hline Liver tumorectomy & $35(100.00)$ \\
\hline 1 time & $24(68.57)$ \\
\hline 2 times & $9(25.71)$ \\
\hline 3 times & $3(8.57)$ \\
\hline Lung metastasectomy & $19(54.29)$ \\
\hline 1 time & $10(28.57)$ \\
\hline 2 times & $3(8.57)$ \\
\hline 3 times & $5(14.29)$ \\
\hline 4 times & $1(2.86)$ \\
\hline \multicolumn{2}{|l|}{ Surgery on other organs } \\
\hline Inferior vena cava tumor thrombectomy & $3(8.57)$ \\
\hline Right atrial tumor thrombectomy & $2(5.71)$ \\
\hline Portal vein tumor thrombectomy & $1(2.86)$ \\
\hline Mediastinal tumorectomy & $2(5.71)$ \\
\hline Intracranial tumorectomy & $3(8.57)$ \\
\hline Spinal and adrenal tumorectomy & $1(2.86)$ \\
\hline \multicolumn{2}{|l|}{ Recurrence and progression } \\
\hline Liver recurrence & $14(40.00)$ \\
\hline Lung recurrence & $18(51.42)$ \\
\hline Head recurrence & $11(31.43)$ \\
\hline Mediastinal recurrence & $1(2.86)$ \\
\hline Progression after liver tumorectomy & $3(8.57)$ \\
\hline \multicolumn{2}{|l|}{ Prognosis } \\
\hline $\mathrm{CR}$ & $7(20.00)$ \\
\hline PR & $2(5.71)$ \\
\hline Dead & $24(68.57)$ \\
\hline Censored & $3(8.57)$ \\
\hline
\end{tabular}

$\mathrm{CR}$, complete remission; PR, partial remission.

metastasectomy, 5 (14.29\%) patients underwent 3 times lung metastasectomy, and $1(2.86 \%)$ patient underwent 4 times lung metastasectomy.

Patients were followed up to December 2018, with a 

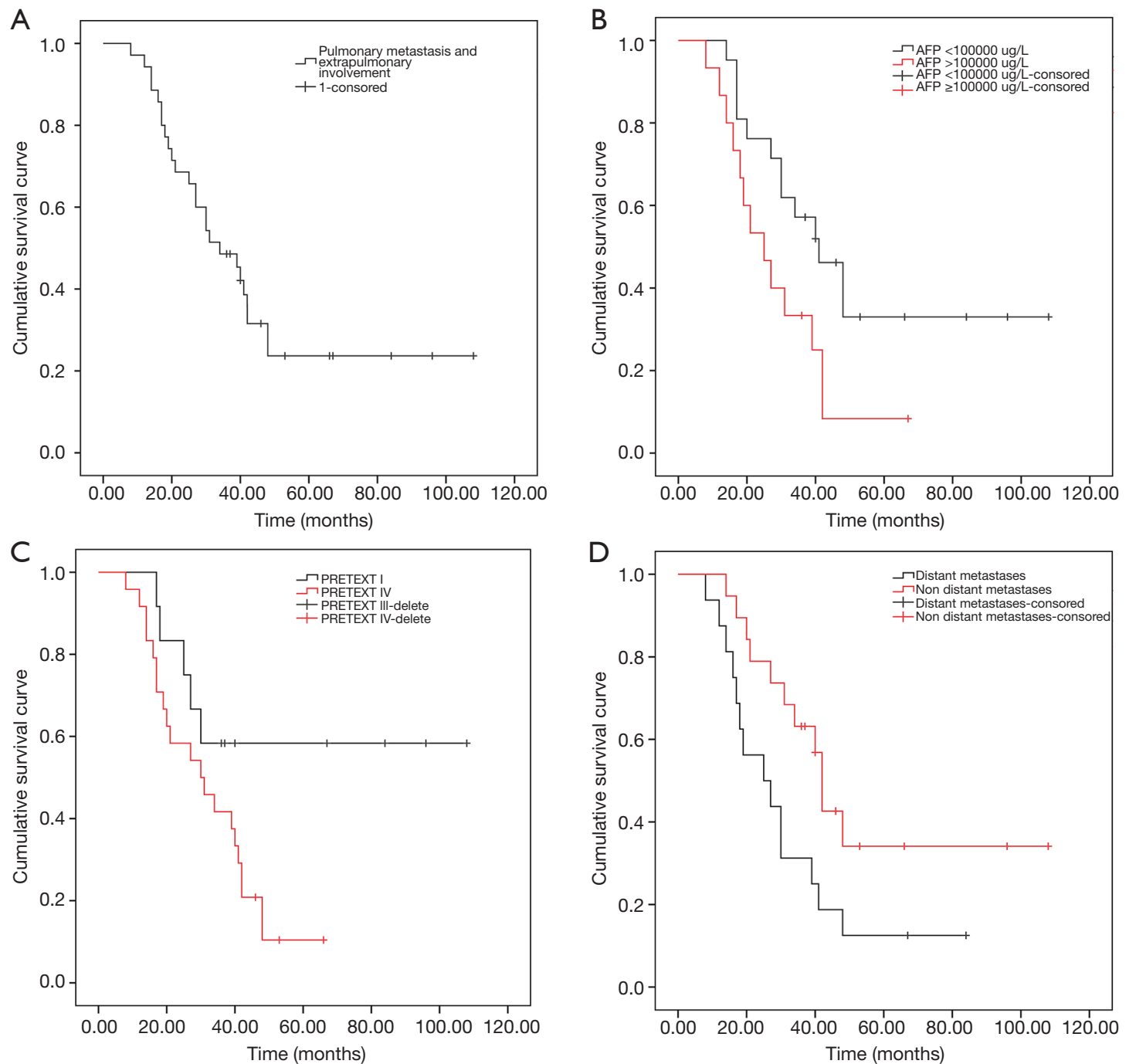

Figure 4 Survival curves of HB children. (A) Overall survival curve of all patients with pulmonary metastasis and extrapulmonary involvement. (B) Kaplan-Meier survival curves according to serum AFP (AFP <100,000 $\mu \mathrm{g} / \mathrm{L}$ and AFP $\geq 100,000 \mu \mathrm{g} / \mathrm{L}$ ). (C) Kaplan-Meier survival curves according to PRETEXT stage (stage III and stage IV). (D) Kaplan-Meier survival curves according to distant metastases (distant metastases and non-distant metastases). AFP, alpha-fetoprotein.

median follow-up of 32.5 months. At the study closing date, 9 patients were alive, 24 patients had died, and 3 patients were censored (died from non-tumor causes). In addition, $14(40.00 \%)$ patients had liver recurrence, 18 (51.42\%) patients had lung recurrence, 11 (31.43\%) patients had head recurrence, and $1(2.86 \%)$ patient had mediastinal recurrence.

\section{Survival analysis}

The survival curve of all patients was shown in Figure $4 A$.
The median OS was 32.5 months (range, 8-108). For 35 patients receiving standardized chemotherapy, the survival time was $47.16 \pm 6.33$ months, the 3 -year OS rate was $48.6 \%$, and the 5 -year OS rate was $23.7 \%$. For 1 patient who only received three cycles of chemotherapy after surgery, the survival time was 17 months.

Considering the level of serum AFP (Figure 4B), the OS of patients with serum AFP $<100,000 \mu \mathrm{g} / \mathrm{L}$ was longer than that of patients with AFP $\geq 100,000 \mu \mathrm{g} / \mathrm{L}(56.21 \pm 8.68 v s$. $28.57 \pm 4.19$ months, $\left.\chi^{2}=4.511, P=0.034\right)$. PRETEXT stage III was associated with a longer disease specific survival (OS, 

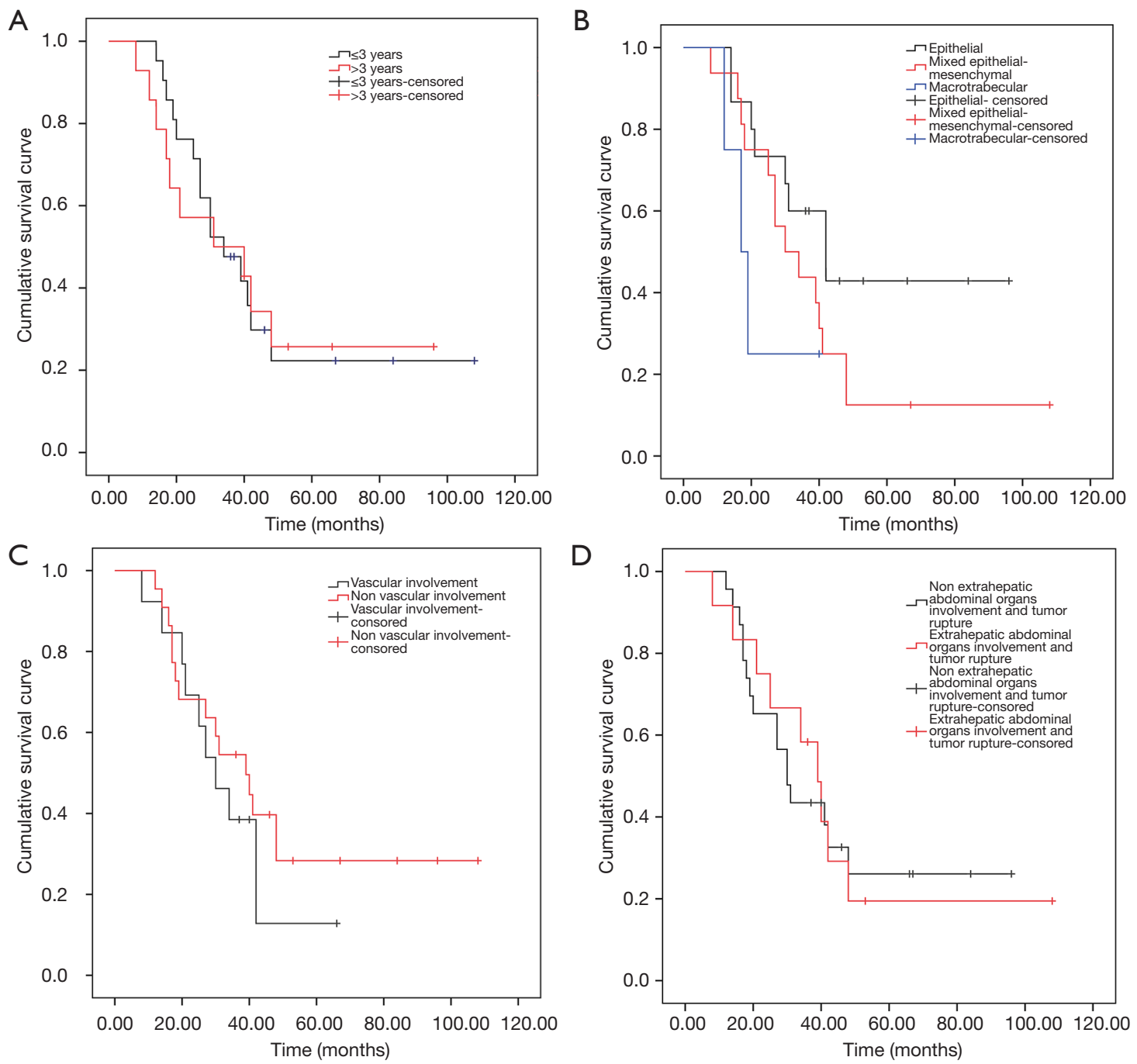

Figure 5 Overall survival according to different categories. (A) Kaplan-Meier survival curves according to age group (age $\leq 3$ and $>3$ years). (B) Kaplan-Meier survival curves according to pathology type (epithelial, macrotrabecular and mixed type). (C) Kaplan-Meier survival curves according to vascular involvement (vascular involvement and non-vascular involvement). (D) Kaplan-Meier survival curves according to extrahepatic abdominal organs involvement (extrahepatic abdominal organs involvement and non-extrahepatic abdominal organs involvement).

$72.75 \pm 12.08$ months) compared with PRETEXT stage IV (OS, 32.04 \pm 3.44 months) $\left(\chi^{2}=4.148, \mathrm{P}=0.042\right)$ (Figure $4 C$ ). In addition, the OS for patients with distant metastases (brain and bone metastasis) and without distant metastases were $32.00 \pm 5.60$ and $58.05 \pm 9.34$ months, respectively $\left(\chi^{2}=4.620, \mathrm{P}=0.032\right)$ (Figure $\left.4 D\right)$.

No statistically significant differences in OS were identified when the study group was divided by age (age $\leq 3$ and $>3$ years, $47.27 \pm 7.98$ vs. $43.90 \pm 8.99$ months, $\chi^{2}=0.002$, $\mathrm{P}=0.963$ ), or by pathology type (epithelial, macrotrabecular and mixed type, $57.01 \pm 9.15$ vs. $39.63 \pm 7.04$ vs. $22.00 \pm 5.35$ months, $\chi^{2}=4.166, \mathrm{P}=0.125$ ), or by vascular involvement (vascular involvement and non-vascular involvement, $33.00 \pm 4.82$ vs. $51.13 \pm 8.23$ months, $\chi^{2}=0.659, \mathrm{P}=0.417$ ) (Figure $5 A, B, C)$. In addition, there was no statistical difference in OS between patients with extrahepatic abdominal organs involvement and tumor rupture and patients without extrahepatic abdominal organs involvement and tumor rupture $(44.77 \pm 6.98 v s .45 .93 \pm 9.83$ months, $\left.\chi^{2}=0.002, \mathrm{P}=0.966\right)$ (Figure 5D). 


\section{Discussion}

$\mathrm{HB}$ is the most common primary hepatic tumor in pediatric population. The incidence of $\mathrm{HB}$ has been increasing in the past 30 years, with an annual increase of up to $2.7 \%$ (20), which may be related to the improved survival rate in premature and very low birth weight infants (19). Although treatment outcome has improved over the past two decades, the presence of distant metastasis at the time of diagnosis is still the strongest predictor of poor prognosis $(15,23)$. Lung is the most common site of distant metastasis of $\mathrm{HB}$, and there are few reports on the prognosis in $\mathrm{HB}$ patients with pulmonary metastasis and extrapulmonary involvement (13). In this study, we aimed to analyze the clinical characteristics and outcome in $\mathrm{HB}$ children with pulmonary metastasis and extrapulmonary involvement.

Zhang et al. retrospectively analyzed $102 \mathrm{HB}$ patients from September 2006 to June 2014, and reported that 49 (48.04\%) HB patients had distant metastasis (25). Among these 49 patients, 37 (75.51\%) had lung metastasis, $10(20.41 \%)$ had vascular metastasis, 17 (34.69\%) had intrahepatic metastasis, and $6(12.24 \%)$ had bone metastasis. These results indicated that lung was the most common site of distant metastasis of HB. In addition, Zsiros et al. reported that the patients with vascular involvement and extrahepatic abdominal organs involvement accounting for $39 \%$ and $10 \%$, respectively (26). In this study, we retrospectively analyzed $36 \mathrm{HB}$ children with pulmonary metastasis and extrapulmonary involvement, and found that $16(44.44 \%)$ cases had vascular metastasis, 13 (36.11\%) cases had brain metastasis, and $10(27.78 \%)$ cases had extrahepatic abdomen involvement. These results indicated that the common sites of $\mathrm{HB}$ with extrapulmonary metastasis were blood vessels, brain, and extrahepatic abdominal organs, which consistent with previous reports.

Previous studies had demonstrated that the 5-year survival rate of $\mathrm{HB}$ with lung metastasis was about $25-50 \%$ $(19,20)$. These results were found to be higher than in our study. In this study, the median survival time of 35 patients who received standardized chemotherapy for more than 6 cycles was $47.16 \pm 6.33$ months, with a 3 -year survival rate of $48.6 \%$ and 5 -year survival rate of $23.7 \%$. The possible reason was that we included $\mathrm{HB}$ patients with pulmonary metastasis and extrapulmonary involvement, which had a relatively poor prognosis.

Previous studies showed that the prognostic factors of HB included distant metastasis, vascular involvement, extrahepatic abdominal organs involvement and tumor rupture, AFP level and PRETEXT stage $(15,27)$. In this study, we found that AFP level, PRETEXT stage and distant metastases had significant impact on survival time, which consistent with previous reports. However, there no statistically significant differences in OS were identified when the study group was divided by vascular involvement or extrahepatic abdominal organs involvement. Considering lung metastasis itself was the main factor affecting prognosis, and the sample size was not large enough, vascular involvement or extrahepatic abdominal organs involvement may have no significant effect on the outcome.

\section{Conclusions}

The common sites of extrapulmonary metastasis of $\mathrm{HB}$ were blood vessels, brain and extrahepatic abdominal organs. The overall prognosis of HB patients with lung metastasis and extrapulmonary involvement was poor, especially those with PRETEXT stage IV, high AFP level or distant metastases. These results may provide some valuable information for further treatment.

\section{Acknowledgments}

Funding: This work was supported by "Climbing the Peak" Talent Plan of Beijing Municipal Administration of Hospital [number DFL20180201].

\section{Footnote}

Reporting Checklist: The authors have completed the STROBE reporting checklist. Available at http://dx.doi. org/10.21037/tcr-20-1876

Data Sharing Statement: Available at http://dx.doi. org/10.21037/tcr-20-1876

Conflicts of Interest: All authors have completed the ICMJE uniform disclosure form (available at http://dx.doi. org/10.21037/tcr-20-1876). The authors have no conflicts of interest to declare.

Ethical Statement: The authors are accountable for all aspects of the work in ensuring that questions related to the accuracy or integrity of any part of the work are appropriately investigated and resolved. The study was conducted in accordance with the Declaration of Helsinki (as revised in 2013). The study was approved by the ethics 
committee of the Beijing Tongren Hospital, Capital Medical University (No.: 20180212) and informed consent was taken from all participants and their guardians.

Open Access Statement: This is an Open Access article distributed in accordance with the Creative Commons Attribution-NonCommercial-NoDerivs 4.0 International License (CC BY-NC-ND 4.0), which permits the noncommercial replication and distribution of the article with the strict proviso that no changes or edits are made and the original work is properly cited (including links to both the formal publication through the relevant DOI and the license). See: https://creativecommons.org/licenses/by-nc-nd/4.0/.

\section{References}

1. Sharma D, Subbarao G, Saxena R. Hepatoblastoma. Semin Diagn Pathol 2017;34:192-200.

2. Towbin AJ, Braojos Braga FDC, Zhang B, et al. Fractures in children with newly diagnosed hepatoblastoma. Pediatr Radiol 2018;48:581-5.

3. McCarville MB, Roebuck DJ. Diagnosis and staging of hepatoblastoma: imaging aspects. Pediatr Blood Cancer 2012;59:793-9.

4. Vlajnic T, Brisse HJ, Aerts I, et al. Fine needle aspiration in the diagnosis and classification of hepatoblastoma: Analysis of 21 New Cases. Diagn Cytopathol 2017;45:91-100.

5. Fuchs J, Rydzynski J, Von Schweinitz D, et al. Pretreatment prognostic factors and treatment results in children with hepatoblastoma: a report from the German Cooperative Pediatric Liver Tumor Study HB 94. Cancer 2002;95:172-82.

6. Aronson DC, Schnater JM, Staalman CR, et al. Predictive value of the pretreatment extent of disease system in hepatoblastoma: results from the International Society of Pediatric Oncology Liver Tumor Study Group SIOPEL-1 study. J Clin Oncol 2005;23:1245-52.

7. Meyers RL, Rowland JR, Krailo M, et al. Predictive power of pretreatment prognostic factors in children with hepatoblastoma: a report from the Children's Oncology Group. Pediatr Blood Cancer 2009;53:1016-22.

8. Maibach R, Roebuck D, Brugieres L, et al. Prognostic stratification for children with hepatoblastoma: the SIOPEL experience. Eur J Cancer 2012;48:1543-9.

9. Hermann RE, Lonsdale D. Chemotherapy, radiotherapy, and hepatic lobectomy for hepatoblastoma in an infant: report of a survival. Surgery 1970;68:383-8.
10. Tiao GM, Bobey N, Allen S, et al. The current management of hepatoblastoma: a combination of chemotherapy, conventional resection, and liver transplantation. J Pediatr 2005;146:204-11.

11. Towu E, Kiely E, Pierro A, et al. Outcome and complications after resection of hepatoblastoma. J Pediatr Surg 2004;39:199-202; discussion 199-202.

12. Honeyman JN, La Quaglia MP. Malignant liver tumors. Semin Pediatr Surg 2012;21:245-54.

13. Hishiki T, Matsunaga T, Sasaki F, et al. Outcome of hepatoblastomas treated using the Japanese Study Group for Pediatric Liver Tumor (JPLT) protocol-2: report from the JPLT. Pediatr Surg Int 2011;27:1-8.

14. Shi Y, Geller JI, Ma IT, et al. Relapsed hepatoblastoma confined to the lung is effectively treated with pulmonary metastasectomy. J Pediatr Surg 2016;51:525-9.

15. Meyers RL, Maibach R, Hiyama E, et al. Risk-stratified staging in paediatric hepatoblastoma: a unified analysis from the Children's Hepatic tumors International Collaboration. Lancet Oncol 2017;18:122-31.

16. Rai P, J HF. Cerebral metastasis of hepatoblastoma: a review. J Pediatr Hematol Oncol 2016;38:279-82.

17. Rowland JM. Hepatoblastoma: assessment of criteria for histologic classification. Med Pediatr Oncol 2002;39:478-83.

18. Roebuck DJ, Aronson D, Clapuyt P, et al. 2005 PRETEXT: a revised staging system for primary malignant liver tumours of childhood developed by the SIOPEL group. Pediatr Radiol 2007;37:123-32; quiz 249-50.

19. Brown J, Perilongo G, Shafford E, et al. Pretreatment prognostic factors for children with hepatoblastoma-results from the International Society of Paediatric Oncology (SIOP) study SIOPEL 1. Eur J Cancer 2000;36:1418-25.

20. Perilongo G, Brown J, Shafford E, et al. Hepatoblastoma presenting with lung metastases: treatment results of the first cooperative, prospective study of the International Society of Paediatric Oncology on childhood liver tumors. Cancer 2000;89:1845-53.

21. Meyers RL, Tiao G, de Ville de Goyet J, et al. Hepatoblastoma state of the art: pre-treatment extent of disease, surgical resection guidelines and the role of liver transplantation. Curr Opin Pediatr 2014;26:29-36.

22. Perilongo G, Maibach R, Shafford E, et al. Cisplatin versus cisplatin plus doxorubicin for standard-risk hepatoblastoma. N Engl J Med 2009;361:1662-70.

23. Zsiros J, Maibach R, Shafford E, et al. Successful treatment 
of childhood high-risk hepatoblastoma with dose-intensive multiagent chemotherapy and surgery: final results of the SIOPEL-3HR study. J Clin Oncol 2010;28:2584-90.

24. Committee CA-CAP, Group CMAPO-s. Expert consensus for multidisciplinary Management of Hepatoblastoma (CCCG-HB-2016). Chinese Journal of Pediatric Surgery 2017;38:733-9.

25. Zhang $\mathrm{Y}$, Zhang $\mathrm{W}$, Tang $\mathrm{S}$, et al. A single-center

Cite this article as: Hu HM, Zhang WL, Wang YZ, Zhang Y, Yi Y, Li F, Zhi T, Huang DS. Treatment outcomes for hepatoblastoma children with pulmonary metastasis and extrapulmonary involvement: experience of 36 cases at a single institution. Transl Cancer Res 2020;9(10):6402-6411. doi: $10.21037 /$ tcr-20-1876 retrospective study of pediatric hepatoblastoma. Oncol Lett 2016;12:3919-25.

26. Zsiros J, Brugieres L, Brock P, et al. Dose-dense cisplatinbased chemotherapy and surgery for children with highrisk hepatoblastoma (SIOPEL-4): a prospective, singlearm, feasibility study. Lancet Oncol 2013;14:834-42.

27. Hiyama E. Pediatric hepatoblastoma: diagnosis and treatment. Transl Pediatr 2014;3:293-9. 